\title{
Contrasting Reaction Modality between Electrochemical Sodiation and Lithiation in NiO Conversion Electrode Materials
}

\author{
Kai $\mathrm{He}^{1}$, Feng $\mathrm{Lin}^{2}$, Eric A. Stach ${ }^{1}$, Yifei Mo ${ }^{3}$, Huolin L. Xin ${ }^{1}$ and Dong $\mathrm{Su}^{1}$ \\ 1. Center for Functional Nanomaterials, Brookhaven National Laboratory, Upton, NY 11973 \\ 2. Environmental Energy Technologies Division, Lawrence Berkeley National Laboratory, Berkeley, \\ CA, 94720 \\ 3. Department of Materials Science and Engineering, University of Maryland, College Park, MD, 20742
}

The diverse requirements of energy storage devices in different applications call for advancements not only in lithium ion chemistry but also in new battery chemistries. Specifically, renewed interest in sodium ion batteries arises from their potential to provide a sustainable and low-cost solution for largescale energy storage such as electric vehicles and power grids [1]. Although the lithium ion battery electrodes can possibly adapt to the sodium ion technology, the dissimilarities between lithium and sodium characteristics may strongly affect the electrochemical processes and the overall battery performance $[2,3]$. Prior studies on nickel oxide $(\mathrm{NiO})$ as a conversion electrode material for lithium ion batteries have uncovered the lithiation mechanism through the heterogeneous surface-to-bulk reaction pathways [4]. Therefore, identifying the dynamic sodiation process in the same electrode material and comparing it with lithiation to understand the underlying reaction mechanism is of fundamental importance.

Here, we present the real-time observation of the sodiation process in $\mathrm{NiO}$ nanosheets using in-situ transmission electron microscopy (TEM). The in-situ nano-battery was set up in a dry cell configuration within TEM (Figure 1a) and further correlated to ex-situ electron and synchrotron X-ray spectroscopies of coin cells. We have integrated the in situ TEM imaging, diffraction, spectroscopy, and tomography (Figure 1a-e), to draw a full picture of the entire sodiation reaction at all relevant length scales.

The structural and morphological evolutions during both sodiation and lithiation were in-situ tracked, as shown in Figure 1a. In contrast to the heterogeneous lithiation, in which the lithium conversion starts from the surface of pristine $\mathrm{NiO}$ and penetrates into the interior redox sites via the lithiation "fingers", the sodiation proceeds conformally from surface redox sites toward the inner bulk, but still leaving most of interior region unreacted after 960 s. The reduced $\mathrm{Ni}$ nanoparticles precipitate from the $\mathrm{NiO}$ lattice and coherently attach on the surface, as revealed by the aberration-corrected scanning transmission electron microscopy (STEM) image in Figure 1b. The phase transformation of $\mathrm{NiO}+\mathrm{Na} \rightarrow \mathrm{Ni}+\mathrm{Na}_{2} \mathrm{O}$ has been confirmed by the in-situ electron diffraction (Figure 1c), while the degree of the phase conversion is only in a limited amount. The electron energy-loss spectroscopy (EELS) mapping of Ni valance states (Figure 1d) indicates that only surface redox sites were reacted with sodium to form Ni nanoparticles, which can also be justified by three-dimensional visualization using electron tomography (Figure 1e). All these microscopic results consistently show a surface-dominant sodiation reaction modality distinct from the lithiation, which is also reproduced and explained by the molecular dynamics calculation. In summary, the fundamental understanding obtained by this in-situ methodology has contrasted the difference in reaction mechanisms between sodium and lithium chemistries and may provide valuable implications to future advances of sodium ion batteries [5]. 
References:

[1] M D Slater et al, Adv. Func. Mater. 23 (2013) 947.

[2] Y Wen, K He et al, Nature Commun. 5, (2014) 4403.

[3] K He et al, ACS Nano 8, (2014) 7251.

[4] K He et al, Nano Lett. 15, (2015) 1437.

[5] The authors acknowledge funding support from U.S. Department of Energy, Office of Basic Energy Sciences, under Contract Number DE-AC02-98CH10886 and DE-SC-00112704.

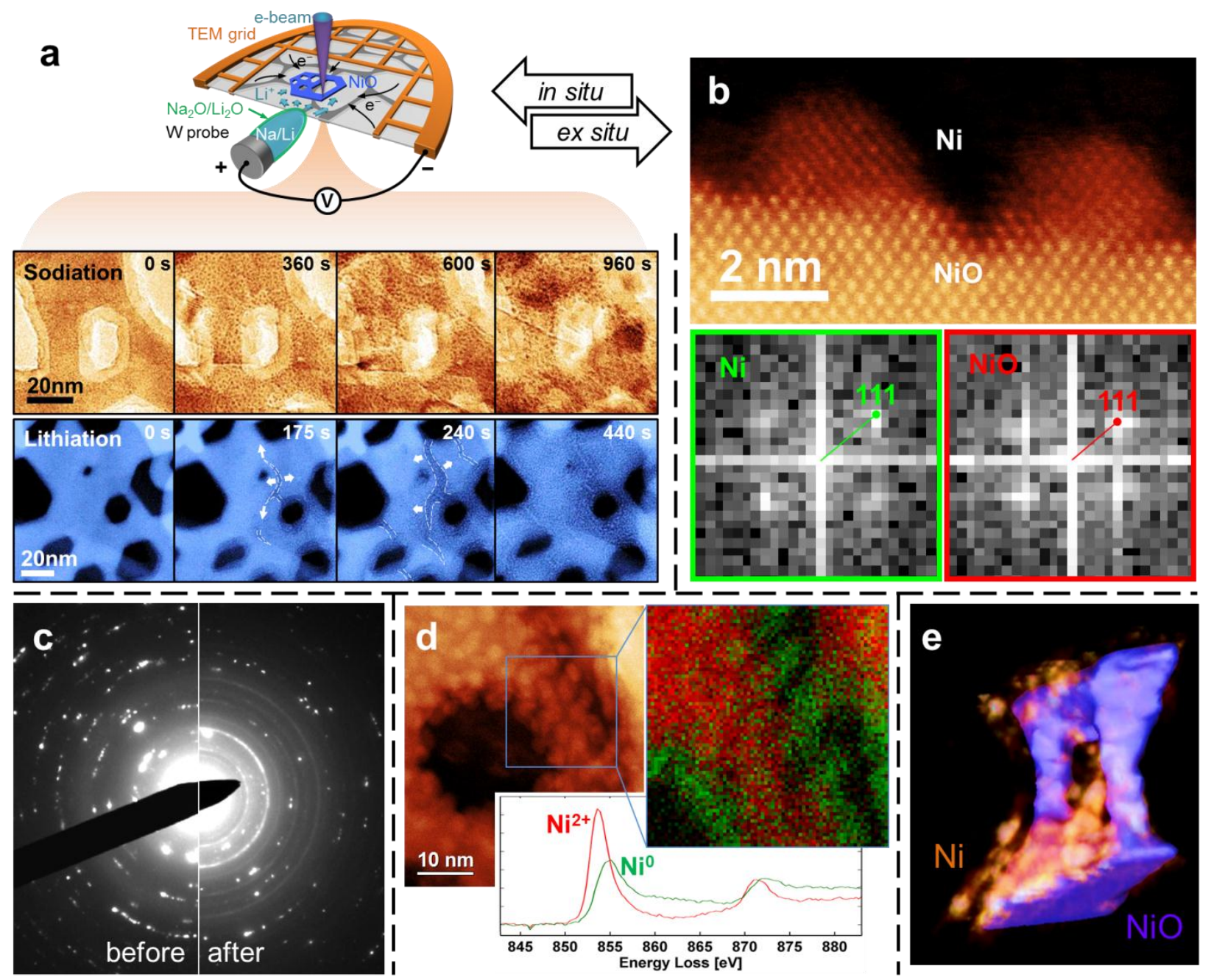

Figure 1. (a) Schematics of in-situ dry cell setup and time-sequenced TEM/STEM image series showing structural evolution during in-situ sodiation and lithiation, respectively. (b) High-resolution STEM image of sodiated sample showing Ni nanoparticles coherently attached on the surface of NiO. The FFT showing the shrinkage of Ni lattice as reduced from NiO. (c) In-situ electron diffraction patterns before and after sodiation. (d) STEM-EELS mapping of Ni valance states and (e) 3D electron tomography showing reduced metallic Ni nanoparticles on the surface of $\mathrm{NiO}$. 